\title{
ASPECTOS MACROSCÓPICOS E HISTOLÓGICOS DA MUCOSA GÁSTRICA DE RATOS WISTAR E SUA UTILIZAÇÃO EM MODELO DE ÚLCERAS GÁSTRICAS
}

\author{
Camilo Amaro de Carvalho ${ }^{1}$, Kenner Moraes Fernandes ${ }^{2}$, \\ Sérgio Luis Pinto da Matta ${ }^{2}$, Claudio César Fonseca ${ }^{2}$, Rogério Pinto ${ }^{1}$, \\ Leandro Licursi de Oliveira ${ }^{2}$ \\ 1 União de Ensino Superior de Viçosa - UNIVIÇOSA - camiloamaro@yahoo.com.br \\ 2 Universidade Federal de Viçosa
}

\begin{abstract}
RESUMO: A busca de novos fármacos e o uso de experimentação animal tem contribuído sobremaneira para o desenvolvimento da saúde, da ciência e tecnologia, promovendo ao longo dos anos a descoberta de medidas profiláticas e tratamentos de enfermidades que acometem os seres humanos. Animais de várias espécies têm sido utilizados desde os primórdios da civilização para o desenvolvimento científico, mas dentre todos os modelos experimentais aos quais podem ser induzidas úlceras gástricas, o rato Wistar, inquestionavelmente, é o mais utilizado. O objetivo deste trabalho foi realizar uma descrição anatômica e histológica do estômago de ratos Wistar os quais foram descritos. Anatomicamente, o estômago do rato Wistar (Rattus norvergicus) está localizado na cavidade abdominal sob a linha mediana ventral, na porção esquerda, caudal ao fígado. Este órgão pode ser dividido em duas regiões glandular e aglandular. Estas regiões estão separadas pela margem pregueada que faz divisão entre o epitélio estratificado pavimentoso queratinizado que reveste a porção aglandular e o epitélio simples cúbico de revestimento da região glandular. A porção aglandular se estende do esôfago até a margem pregueada, já a região glandular encontrase com o corpo e antro pilórico. A descrição anatômica e histológica, aliada a dados bioquímicos e fisiológicos, são de suma importância na pesquisa científica e na busca de novos fármacos. Deste modo, este trabalho poderá contribuir para o aprimoramento de estudos que visam a utilização de ratos Wistar como modelo experimental de úlceras gástricas.
\end{abstract}

Palavras-chave: anatomia; Rattus norvergicus; patologia veterinária

\section{MACROSCOPIC AND HISTOLOGICAL ASPECTS GASTRIC MUCOSA OF WISTAR RAT MODEL AND ITS USE IN GASTRIC ULCERS}

\begin{abstract}
The search of new drugs and the use of animal experimentation had been contributed excessively to the development of the health, of the science and technology, promoting along the years the discovery of prophylactic measured and treatments of illnesses that affect the human beings. Animals of several species have been used from the origins of the civilization for the scientific development, but among all of the experimental models to the which peptic ulcers can be induced, the rats Wistar, doubtless, is the used. The objective of this work was to accomplish an anatomical and histological description. Anatomically, the stomach of the Wistar rat (Rattus norvegicus) is located in the abdominal cavity in the ventral midline in the left portion, caudal to the liver. This body can be divided into two regions glandular and non glandular. These regions are separated by the folded edge that makes the division between the keratinized stratified squamous epithelium that lines the portion of exfoliation and the simple cubic epithelium lining the glandular stomach. The exfoliation portion extending from the esophagus to the bank folded, the glandular region already meets the body and pyloric antrum. The anatomical and histological description, combined with biochemical and physiological, are extremely important in scientific research and the search for new drugs. Thus, this work will contribute to the improvement of studies aimed at the use of rats as an experimental model of gastric ulcers.
\end{abstract}

Key Words: anatomy; Rattus norvergicus; veterinary pathology 


\section{INTRODUÇÃO}

A pesquisa experimental com animais de laboratório tem sido à base do conhecimento e da evolução das ciências biológicas (morfologia, fisiologia, farmacologia), pois possibilitam os ensaios terapêuticos. Os resultados desses ensaios devem ser avaliados criteriosamente e são muitas vezes aplicados em seres humanos (Chorilli, 2007).

Bernard (1865), em seus estudos de fisiologia, lançou os princípios do uso de animais como modelo de estudo com transposição para a fisiologia humana. Seu trabalho "Introdução ao Estudo da Medicina Experimental" procurou estabelecer as regras e os princípios para o estudo experimental da medicina. A idéia de utilização de animais em pesquisas surgiu devido à necessidade do homem em entender patologias ainda não compreendidas, assim como prover novas alternativas terapêuticas (Fagundes e Taha, 2004).

O desenvolvimento da pesquisa experimental em animais de laboratório tem contribuído nos diferentes campos científicos e vem promovendo ao longo dos anos a descoberta de medidas profiláticas e tratamentos de inúmeras enfermidades que acometem os seres vivos. Como exemplos de contribuições científicas advindas de estudos realizados em animais, têm-se a descoberta da insulina, o desenvolvimento de vacinas contra diversas doenças, a produção de soros, o uso terapêutico de antibióticos e o tratamento de vários distúrbios do aparelho digestório, como por exemplo, o desenvolvimento de produtos para o tratamento de úlceras gástricas (Fagundes e Taha, 2004; Andrade, 2006).

Nos últimos 20 anos, observou-se um avanço significativo no estudo de doenças relacionadas às úlceras, gástrica e duodenal. Isto se deve, espe- cialmente, ao desenvolvimento de várias técnicas que têm possibilitado uma observação mais detalhado da mucosa gástrica (Brzozowski, 2003).

O risco de complicação por úlcera tem aumentado em média, resultando 1,25 hospitalizações adicionais para cada 100 pacientes por ano (Almeida, 2004). A hipersecreção ácida é o aspecto mais importante da função gástrica estudado na prática clínica. A influência de alterações nessa função tem sido considerada no desenvolvimento de drogas para serem utilizadas no tratamento de úlceras gástricas (Pohle e Domschke, 2003).

Mesmo com o progresso de métodos alternativos nos últimos anos (estudos in vitro, cultura de células, softwares etc.), os modelos animais ainda se apresentam como opção mais vantajosa por possibilitarem 0 fornecimento de informações sobre o organismo como um todo, o que não é conseguido com outros métodos (Heywood, 1987; Ribeiro et al., 1995; Salén, 1995; Snitkoff, 2004). Dentre todos os modelos experimentais aos quais podem ser induzidas úlceras gástricas, o rato Wistar, inquestionávelmente, é o mais intensamente utilizado. No entanto, é escasso o acervo bibliográfico enfocando aspectos histológicos e anatômicos do estômago de ratos, em especial de ratos Wistar. Portanto, este trabalho teve por objetivo descrever os aspectos macroscópicos e histológicos da mucosa gástrica de ratos Wistar, visando acrescentar conhecimentos sobre a espécie e permitir maior acesso à comunidade científica no uso desta espécie em modelos para estudos de úlceras gástricas.

\section{MATERIAL E MÉTODOS}

Os animais foram manipulados de acordo com o Manual sobre Cuidados e 
Usos de Animais de Laboratório National Research Council (2003) e em concordância com os Princípios Éticos para o uso de Animais de Laboratório preconizados pelo COBEA - Colégio Brasileiro de Experimentação Animal, sendo aprovado pela Comissão de Ética do Departamento de Veterinária da UFV, sob protocolo ํㅜ 44/2008..

Foram utilizados cinco ratos Wistar machos (Rattus norvergicus), adultos com idades de 120 dias, pesando em média 340 gramas e provenientes do Biotério Central do Centro de Ciências Biológicas e da Saúde (CCBS) da Universidade Federal de Viçosa. Os animais foram pesados e alojados em gaiolas individuais, sendo mantidos em condições de temperatura e umidade ambiente controlados e no ciclo claroescuro de 12h (07:00-19:00), no Laboratório de Experimentação Animal do Departamento de Nutrição. Água e ração foram disponíveis ad libitum, sendo retirada a ração $24 \mathrm{~h}$ antes da eutanásia, mantendo-se livre o acesso à água.

Avaliação macroscópica das lesões gástricas

Após remoção do estômago, uma incisão foi realizada ao longo da sua curvatura maior. $\mathrm{O}$ órgão foi lavado com solução de $\mathrm{NaCl}(0,9 \%)$ para retirada de restos alimentares e em seguida fixado em solução de formol neutro a $10 \%$ tamponado, por 24h. O órgão foi analisado em estereomicroscópio Olympus ${ }^{\circledR} \quad$ SZ40, onde foram selecionados os fragmentos contendo ulcerações.

\section{Avaliação microscópica das lesões gástricas}

Os fragmentos selecionados foram destinados ao estudo sob microscopia de luz, e submetidos às técnicas histológicas rotineiras, desde a desidratação em série crescente de alcoóis $70,80,90$ e $100 \%$, até a inclusão em parafina histológica purificada (PF 56-58 $\stackrel{\circ}{ } \mathrm{C}$ ).

Os blocos foram seccionados em micrótomo de rotação manual (Leica ${ }^{\circledR}$ 2045), com espessura de $4 \mu \mathrm{m}$ e os cortes teciduais corados pelas técnicas de Hematoxilina-Eosina (HE) (Grimaldi Filho, 1981).

A análise histológica foi realizada no Laboratório de Biologia Estrutural do Departamento de Biologia Geral da Universidade Federal de Viçosa, em microscópio de luz Olympus ${ }^{\oplus}$ BX 41. Formam obtidas imagens em fotomicroscópico Olympus ${ }^{\circledR}$ C31.

\section{RESULTADOS E DISCUSSÃO}

Anatomicamente, o estômago do rato Wistar está localizado na cavidade abdominal sob a linha mediana ventral, na porção esquerda, caudal ao fígado, com a forma de um C e com uma concavidade cranial, onde se observa a penetração do esôfago. $O$ peritônio encontra-se ligado ao estômago por uma membrana denominada omento maior. Estes dados estão de acordo com as observações realizadas por Freiburg (2007), onde o mesmo ainda relata que o estômago pode ser divido em várias regiões (Figura 1a).

O estômago dos ratos Wistar (Rattus norvergicus) é curto em relação ao segmento intestinal. A cárdia, região que guarnece a entrada do estômago, e o piloro, esfíncter localizado na saída do mesmo, se localizam próximos devido ao formato do órgão. Segundo Sharp e Regina (1998) o estômago apresenta dorsalmente a região do fundo gástrico, aglandular. As demais regiões do estômago (glandulares) estão separadas pela margem pregueada (margo plicatus) e se encontram com o corpo e antro pilórico. Andrew e Hickman (1974) relatam que a porção aglandular é opaca e possui uma coloração esbranquiçada, enquanto a região glandular é translúcida e apre- 




Região Aglandular

1 Esôfago e Região cárdia

2 Curvatura menor

3 Curvatura maior

4 Saco cego

5 Região Fúndica

6 Corpo do estômago

7 Região pilórica

8 Piloro


Figura 1 - Regiões do estômago de ratos Wistar. (A) Esquema representativo do estômago e suas subdivisões (Adaptado de Freiburg, 2007). (B) Visão macroscópica do estômago seccionado ao longo da curvatura maior, expondo a mucosa gástrica. (C) Detalhe da região glandular e aglandular. Margem pregueada (seta); região glandular $\left({ }^{*}\right)$ e região aglandular (ponta de seta).

senta-se com coloração róseoavermelhada, 0 que pode ser confirmado nas análises macroscópicas do órgão (Figura 1b). Porém, o tempo de exposição ao agente fixador pode levar ao clareamento da mucosa glandular.

A margem pregueada é a "linha" de divisão entre o epitélio estratificado pavimentoso queratinizado que reveste a porção aglandular e o epitélio simples cúbico que reveste a região glandular (Figura 1c). Segundo Sharp e Regina (1998) a região glandular possui alta atividade produtora de muco, responsável pela proteção da mucosa gástrica. Dani e Castro (1993) relataram ainda que as glândulas gástricas possuam alta atividade protetora da parede gástrica contra as agressões alimentares e suas próprias excreções glandulares. Esta proteção pode ser atribuída à alta velocidade das mitoses deste epitélio e, também, ao muco. Estes dados permitem inferir que a região glandular é menos acometida pela formação de lesões, em relação à 
região aglandular, devido às propriedades protetoras da mucosa.

Segundo Goodman e Gilman (2006) dentre os fatores protetores estão o muco e bicarbonato produzidos pelas células epiteliais superficiais, após um sinal estimulador originado por prostaglandinas (PG's). Estes lipídeos são sintetizados pelas ciclooxigenases (COX) e possuem afinidade por receptores $\mathrm{EP}_{3}$ para PG's na porção basal das células epiteliais superficiais da mucosa gástrica. $\mathrm{O}$ muco protege a mucosa gástrica de forma mecânica, lubrificando a parede gástrica e prevenindo sua erosão, já o bicarbonato atua como uma proteção química, neutralizando o a acidez proporcionada pelo ácido clorídrico (Andrew e Hickman, 1974; Dani e Castro, 1993).

Segundo Freiburg (2007) a mucosa glandular do estômago de ratos Wistar pode ser dividida em regiões (Figura 1a) com diferentes tipos celulares: região cárdia, região fúndica e região pilórica. Andrew e Hickman (1974) relataram que região cárdia, está localizada ao redor da abertura gástrica do esôfago, se estendendo à borda da margem pregueada. Acredita-se que a mesma seja responsável pela secreção de bicarbonato e muco protetor. A região fúndica é composta pela maior parte da mucosa glandular e ocupa a parte ventral do estômago (curvatura maior). Na região de mucosa oxíntica se localizam as glândulas gástricas com presença de células parietais, que segundo Junqueira e Carneiro (2004) são células responsáveis pela secreção de ácido clorídrico (Figuras 2a e 2b). Estas células se apresentaram com coloração clara e predominam na porção apical e média das glândulas. Além disso, a presença destas células é escassa na base, semelhante às células parietais do estômago humano, conforme descrito por Dani e Castro (1993).

Distribuídas entre as células parietais no colo da glândula, estão localizadas as células mucosas do colo (Figura 2d). Segundo König (2004) estas células secretam um muco, menos viscoso do que o produzido pelas células da mucosa superficial. Foi verificada nestas células uma evidente basofilia que segundo Junqueira e Carneiro (2004) devem-se ao abundante retículo endoplasmático rugoso, que é característico em células produtoras de proteínas e confere a elas coloração azul escuro. Por fim, a região pilórica, que se estende da região fúndica até ao piloro (esfíncter presente entre 0 estômago e o intestino delgado). Segundo Andrew e Hickman (1974) a maior parte das células presentes nas glândulas pilóricas secretam, assim como na região cárdica, muco diretamente no lúmen gástrico, formando uma "cortina" de muco protetor que funciona como uma barreira alcalina e tamponam o $\mathrm{pH}$ ácido do suco gástrico.

As maiores áreas superficiais do estômago estão revestidas por células mucosas superficiais (Figuras 2c e 2d). Dani e Castro (1993) relatam que está células sintetizam muco persistente e espesso, uma característica especial do revestimento do estômago. Estas células apresentaram coloração clara, típica de células produtoras de muco, com núcleo localizado na porção basal. Segundo Junqueira e Carneiro (2004) a presença destas células mucosas e sua secreção são importante para a proteção do epitélio gástrico frente às condições ácidas e da atividade mecânica presente no lúmen.

O epitélio estratificado pavimentoso queratinizado que reveste a região aglandular (Figuras $2 \mathrm{e}$ e $2 \mathrm{f}$ ) se estende do esôfago até a margem pregueada. Esta região é semelhante ao epitélio esofágico humano. Segundo Dani e Castro (1993), o número, a distribuição e o desenvolvimento de glândulas nesta região são muito variáveis no homem e 



Figura 2 - Fotomicrografias das regiões do estômago de ratos Wistar. (A) Região glandular da mucosa gástrica, evidenciando a espessura da mucosa (seta dupla). (B) Mucosa da região glandular evidenciando a muscular da mucosa (MM), túnica submucosa $\left(^{*}\right)$ e a camada muscular (M); (C) Abertura da fosseta gástrica na luz estomacal da região glandular $(F)$ e células mucosas superficiais (seta). (D) Glândulas fúndicas da região glandular, célula parietal ( $P$ ); células zimogênicas (setas); muscular da mucosa (MM). (E) Região aglandular da mucosa gástrica, evidenciando a queratina (Q), mucosa (M), muscular da mucosa (MM), submucosa (SM) e muscular (Mc). (F) Detalhe do epitélio queratinizado da região aglandular, mostrando as camadas epiteliais (seta dupla) e queratina

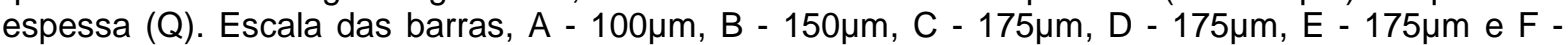
$175 \mu \mathrm{m}$. Coloração HE. 
em outras espécies. Nos cães, estas glândulas são numerosas, porem estão presentes em pequenos números nos macacos e totalmente ausentes nos eqüinos e roedores. No esôfago humano esse tipo de epitélio, é diferenciado para a proteção mecânica, ao contrário da mucosa estomacal que faz mais uma proteção contra substâncias químicas (Dani e Castro, 1993). Entretanto, ainda não se sabe a função da região aglandular no estômago de ratos. Segundo Cunningham (1993), acredita-se que a região aglandular seja um local onde pequena quantidade de digestão fermentativa possa ocorrer. Sugere-se ainda que esteja relacionado com 0 hábito alimentar dos roedores, o que favoreceria uma maior proteção às partículas sólidas ingeridas. Porém, esta região tem menor contato com a ingesta e ainda é considerada a área de maior incidência de formação de úlceras gástricas, especialmente por possuir pouca resistência à ação do ácido clorídrico e pepsina (Andrew e Hickman, 1974; Cunningham, 1993; Sharp e Regina, 1998).

O rato Wistar e a pesquisa experimental com úlceras gástricas

As úlceras pépticas afetam um número considerável de pessoas no mundo (Dani e Castro 1993). A incidência de úlceras é maior nos homens em relação às mulheres $(3: 1)$ e, apesar de ocorrerem em qualquer idade, a úlcera duodenal desenvolve com mais frequência na faixa de 30-55 anos, enquanto a úlcera gástrica na faixa de 50-70 anos (Kumar et al., 2008). Estas dependem de duas condições: presença de ácido e predisposição das mucosas a lesões por fatores diversos (Brzozowski, 2003). Farmacologicamente não há maneiras estabelecidas de interferir com as predisposições, genéticas ou não, da mucosa aos danos (Brzozowski, 2003), sendo o melhor tratamento a prevenção.

Segundo Morini e Grandi (2010) a manutenção da integridade da mucosa gástrica é assegurada por um equilíbrio dinâmico entre os fatores de proteção e nocivas.

Dentre os fatores envolvidos nas lesões da mucosa gástrica encontramse os fatores agressores, como a secreção ácida gástrica e os fatores protetores da mucosa gástrica, como a camada muco-bicarbonato, hidrofobicidade da mucosa e o fluxo sanguíneo (Dani e Castro 1993), sendo a hipersecreção ácida 0 aspecto mais importante da função gástrica estudado na prática clínica. A influência de alterações nessa função tem sido considerada no desenvolvimento de drogas para serem utilizadas no tratamento de úlceras gástricas (Pohle e Domschke, 2003).

O desequilíbrio entre os fatores defensivos e agressivos está na base da formação de erosões/ulcerações ou lesões da mucosa gástrica. A diferença entre uma erosão/lesão e ulceração é que o primeiro se limita à mucosa, enquanto uma ulceração penetra a camada muscular da mucosa. Esta unidade apresenta dois modelos de lesões da mucosa aguda induzida em ratos por agentes gastrotóxicos atuando através de diferentes mecanismos de ação (Morini e Grandi, 2010).

Mediante ao elucidado o desenvolvimento de uma terapêutica adequada para o tratamento das úlceras pépticas tornou-se de vital importância. Segundo Vinagre et al. (2005), as drogas utilizadas atualmente no tratamento de úlceras gástricas não são totalmente eficazes na cicatrização das mesmas. Entre as drogas empregadas, temos antiácidos, anticolinérgicos, antagonistas do receptor $\mathrm{H}_{2}$ para histamina e inibidores de bomba de próton. Entretanto, a maioria destas drogas possui alto valor agregado e vários efeitos adversos. Segundo Yunes 
et al. (2001), faz-se necessário a busca de novos fármacos proporcionando alternativas terapêuticas seguras e de baixo custo e deve ser levado em consideração a grande biodiversidade encontrada no Brasil, que é consequentemente uma rica fonte para novos fármacos. Viegas et al. (2006) relatam que 0 mercado farmacêutico mundial está estimado em 505 bilhões de dólares, em 2004. Estes números exemplificam a importância econômica do setor industrial farmacêutico e, portanto, a dos produtos naturais, de quaisquer origens, como fonte de novos padrões moleculares úteis para descoberta de fármacos. Segundo Simões et al. (2004) muitos achados etnobotânicos tem relatado a grande utilização de plantas e derivados, tanto para o tratamento e prevenção de úlceras gástricas como para outras patologias. Havsteen (2002) em seus estudos têm demonstrado que diferentes substâncias oriundas de plantas, não oferecem apenas gastroproteção, mas também aceleram a cicatrização de úlceras gástricas. Porém, para este tipo de estudo há necessidade de estabelecimento e entendimento dos modelos experimentais utilizados. Assim, a descrição anatômica e histológica, aliada a dados bioquímicos e fisiológicos, são de suma importância na pesquisa científica na busca de novos fármacos. Deste modo, este trabalho poderá contribuir para o aprimoramento de estudos que visam a utilização de ratos Wistar como modelo experimental de úlceras gástricas. Entretnato, novos estudos visando analisar os parâmetros fisiológicos da homestasia no rato Wistar deverão ser realizados, pois o mesmo é muito utilizado em pesquisas experimentais, mas em contrapartida há poucas referências disponíveis, o que dificulta e atrasa os estudos pré-clínicos. Uma maior fonte bibliográfica sobre a espécie favorecerá uma maior produção de produtos terapêuticos e rapidez dos resultados de pesquisas científica utilizando o modelo em questão.

\section{CONCLUSÃO}

A utilização de modelos animais na pesquisa científica é de grande importância para o desenvolvimento da ciência e da humanidade. Porém, há necessidade de um maior número de fontes bibliográficas que relatem sobre os achados histológicos e macrocópicos das espécies estudadas. Assim, a descrição do estômago de ratos Wistar realizada neste trabalho visou acrescentar conhecimentos e permitir maior acesso à comunidade científica que faz uso da espécie como modelo de úlceras gástricas.

\section{REFERÊNCIAS}

ALMEIDA, A.B.A. Atividade anti-ulcerogênica e antiinflamatória intestinal da Arctium lappa. 2004. 147f. Tese (Doutorado em Biologia Funcional e Molecular) - Curso de pósgraduação em Biologia Funcional e Molecular, Universidade Estadual de Campinas.

ANDRADE, M.C.R. A utilização de Símios do gênero Callithrix como modelo experimental. Boletim informativo. Colégio Brasileiro de Experimentação Animal 03-97/98: 5, 1998. Disponível em:

<http://www.cobea.org.br/index.php?p=animais > Acesso em 20/05/2011.

ANDREW, W.; HICKMAN, C. P. Histology of the vertebrates. Saint Louis: The C.V. Mosby Company, 1974. 275 p.

BRZOZOWSKI, T. Experimental production of peptic ulcer, gastric damage and câncer models and their use in pathophysiological studies and pharmacological treatment - Polish achievements. Journal Physiology

Pharmacology, v.54, n.3, p.99-126, 2003.

CHORILLI, M.; MICHELIN, D.C.; SALGADO, H.R.N. Animais de laboratório: o camundongo. Revista de Ciências Farmacêuticas Básica e Apliada, v.28, n.1, p.11-23, 2007.

BERNARD, C. An introduction to the study of experimental medicine (1865). In: Images from the history of medicine division. National Library of Medicine. Disponível em: 
$<\mathrm{http}: / /$ www.nlm.nih.gov/>. Acesso em: 20/05/2011.

CONSELHO FEDERAL DE MEDICINA VETERINÁRIA. Resolução no 714, de 20 de junho de 2002. Dispõe sobre procedimentos e métodos de eutanásia em animais, e dá outras providências. Diário Oficial da União (DOU), 21 de junho de 2002. Disponível em: <http://www.cfmv.org.br/portal/legislacao/resoluc oes/resolucao_714.pdf >. Acesso em: 20/05/2011.

CUNNINGHAM, J.G. Tratado de fisiologia veterinária. 3.ed. Rio de Janeiro: Guanabara Koogan, 2004. 596 p.

DANI, R.; CASTRO, L.P. Gastroenterologia clínica. 3.ed. Rio de Janeiro: Ganabara Koogan, 1993. 1103p.

FAGUNDES, D.J.; TAHA, M.O. Modelo animal de doença: critérios de escolha e espécies de animais de uso corrente. Acta Cirúrgica Brasileira, v.19, n.1, p.59-65, 2004.

FIGUEIREDO, R.D. Lesões gástricas ulcerativas em decorrência do uso de antiinflamatórios não esteroidais. 2006. 31f. Monografia (Especialização em Diagnóstico e Cirurgia de Eqüinos) - Curso de Pós-graduação em Diagnóstico e Cirurgia de Eqüinos, Universidade de Santo Amaro.

FREIBURG, J. L. Abdominale Sonographie bei der Ratte (Rattus norvegicus $f$. domestica). 2007. 131f. Tese (Doutorado em Medicina Veterinária) - Curso de Pós-graduação em Medicina Veterinária, Universität München.

FONSECA, C.C.; PINTO, A.S.; SALCEDO, J.H.P. ET al. Normas de conduta para o uso de animais no ensino, pesquisa e extensão do DVT/UFV. Disponível em:

$<$ http://www.dvt.ufv.br/docs/normas.pdf >. Acesso em: 20 maio 2011.

GOODMAN, A.G.; GILMAN, L.L. As bases farmacológicas da terapêutica. 11.ed. Rio de Janeiro: McGram-Hill Interamericana do Brasil, 2002. $1821 \mathrm{p}$.

GRIMALDI, G. F. Manual de técnica histológica. Rio de Janeiro: Instituto Oswaldo Cruz, $1981.70 \mathrm{p}$.

HAMMOND, C.J.; MASON, D.K.; WATKINS, K.L. Gastric ulceration in mature thoroughbred horses. Equine Veterinary Journal. v.18, n.4, p.284-287, 1986.

HAVSTEEN, B. H. The biochemistry and medical significance of the flavonoids.

Pharmacology \& Therapeutics, v.96, p.67-202, 2002.
HEYWOOD, R. The use of animals in testing. Alternatives to Laboratory Animals, v.14, n.4, p.329-333, 1987.

JUNQUEIRA, L.C. e CARNEIRO, J. Histologia Básica. 10.ed., Rio de Janeiro: Guanabara Koogan, 2004. 488p.

KÖNIG, L.; HORST, E; LIEBICH; H.G. Anatomia dos Animais Domésticos. Porto Alegre: Artmed, v.2, 2004. 420 p.

KUMAR, V.; ABBAS, A. K.; FAUSTO, N. et al. Robbins Patologia Básica. 8.ed., São Paulo: Elsevier, 2008. 1028p.

MORINI, G.; GRANDI, D. Methods to Measure Gastric Mucosal Lesions in the Rat. Current Protocols in Toxicology. v.43, v.21, 2010. Disponível em:

<http://www.ncbi.nlm.nih.gov/pubmed/20967744 >. Acesso em: 20 maio 2011.

NRC. National Research Council. Manual sobre cuidados e usos de animais de laboratório. Goiânia: AAALAC/COBEA, 2003. 75-89 p.

OTOFUJI, G.M. Vias envolvidas no mecanismo de ação do efeito gastroprotetor das raízes da Pfaffia glomerata (Spreng) Pedersen. 2005. 177f. Dissertação (Mestrado em Farmacologia) - Curso de Pós-graduação em Farmacologia, Universidade Federal do Paraná.

POHLE, T; DOMSCHKE, W. Gastric function measurements in drug development. British Journal of Clinical Pharmacology, v.6, n.2, p.156-164, 2003.

RIBEIRO, S.M.L.; CAMPOS, P.; TIRAPEGUI, J. O rato como animal de laboratório: histórico, dados biológicos e análise crítica de seu uso. Revista de Farmácia Bioquímica Universidade de São Paulo, v.31, n.1, p.21-28, 1995.

SALÉN, J.C.W. Animal models: principles and problems. 3.ed. Boston: CRC Press, 1995. 560p.

SHARP, P.E.; LA REGINA, M.C. The Laboratory rat. Washington: CRC Press, 1998. 214p.

SIMÕES, C.O.; SCHENKEL, E. P.; GOSMÃO, G. ET al. Farmacognosia da planta ao medicamento. 5.ed., Porto Alegre/Florianópolis: Editora UFRGS, 2004. 821p.

SNITKOFF, G.G. Testes biológicos. In: GENNARO, A.R. Remington: a ciência e a prática da farmácia. 20.ed. Rio de Janeiro: Guanabara Koogan, 2004, p.556-568.

VIEGAS JUNIOR, C.; BOLZANI, V. S.; BARREIRO, E. J. Os produtos naturais e a 
química medicinal moderna. Química Nova, v.29, n.2, p.326-337, 2006.

VINAGRE, A. M. Efeito anti-ulcerogênico do extrato de Chlorella vulgaris. 2005. $74 f$.

Dissertação (Mestrado em Biologia Funcional e Molecular)-Universidade Estadual de Campinas, Campinas.
YUNES, R.A.; PEDROSA, R.C.; CECHINEL FILHO, V. A necessidade do desenvolvimento da indústria de fitoterápicos e fitofármacos no Brasil. Química Nova. v.24. n.1, p.147-152, 2001.

WERNECK, H. J. L. Guia de dissecação de vertebrados. Belo Horizonte: UFMG, 1966. 86p. 